\title{
ANÁLISIS Y PERSPECTIVAS DE LA ALIANZA DEL PACÍFICO ${ }^{1}$
}

\author{
Jairo Andrés Castaño Peña
}

doi: 10.18543/ed-64(1)-2016pp281-305

Sumario: 1 . Antecedentes. 2. Contexto. 3. Creación, estructura Y ORGANIZACIÓN. 3.1. Objetivos. 3.2. Órganos y funcionamiento. 3.2.1. Cumbres. 3.2.2. Presidencia pro tempore. 3.2.3. El Consejo de Ministros. 3.2.4. El GAN. 3.2.5. Comités y Grupos Técnicos. 3.2.6. Países observadores. 3.2.7. Consejo Empresarial de la Alianza del Pacífico. 4. Avances y logros. 5. Perspectivas. 6. Conclusiones. 7. ReferenCIAS BIBLIOGRÁFICAS. 8. INFORMES DE INSTITUCIONES Y DOCUMENTOS DIFUNDIDOS EN INTERNET.

\section{ANTECEDENTES}

La integración latinoamericana ha sido un anhelo constante de la región que ya veía sus primeras expresiones en la Carta de Jamaica escrita por Simón Bolívar durante el periodo de independencia. Sin embargo, la integración sigue siendo un punto pendiente de la agenda de todos los países latinoamericanos. Si bien es cierto que ha habido múltiples iniciativas con este propósito, también lo es que las diferencias en materia política, económica e incluso cultural han impedido que haya un liderazgo claro que marque el

1 Trabajo realizado en el marco del Proyecto de Investigación Constitución y mercado en la crisis de la integración europea. $\mathrm{I}+\mathrm{D}+\mathrm{i}$ del Programa Estatal de Investigación, Desarrollo e Innovación orientada a los Retos de la Sociedad, del Ministerio de Economía y Competitividad (DER2013-48327-C3-1-R). Investigadores principales: José Luis GARCia Guerrero y María Luz Martínez Alarcón. 
sentido de dicho proceso y que logre una posición común con capacidad de dar fuerza a las diferentes iniciativas.

La integración latinoamericana aparece en las constituciones de Colombia y Perú como un compromiso y un deber del Estado ${ }^{2}$. Las constituciones de Chile y de México, por su parte, no tienen cláusulas que destaquen la importancia de la integración latinoamericana dentro del texto constitucional, pero esto no implica que este silencio deba ser interpretado como un límite para lograrlo. Las cuatro constituciones de los países parte de la Alianza reconocen el valor normativo de los tratados internacionales, especialmente en lo que concierne a la protección de los Derechos Humanos, de ahí la eficacia que ha demostrado el Sistema Interamericano de Protección de los Derechos Humanos $^{3}$. Sin embargo, la integración política y económica lleva un ritmo propio, mucho más lento y sobre todo inestable, ya que depende en buena medida de la voluntad de cada gobernante y de su posición política.

Los proyectos de integración más importantes en Latinoamérica han sido: Comunidad Andina de Naciones CAN (1969), Asociación Latinoamericana De Integración ALADI (1980), en reemplazo de Asociación Latinoamericana de Libre Comercio (ALALC), Mercado Común del Sur MERCOSUR (1991), Alianza Bolivariana para los Pueblos de Nuestra América - Tratado de Libre Comercio ALBA (2004), Unión de Naciones Suramericanas UNASUR (2004), Comunidad de Estados Latinoamericanos y Caribeños CELAC (2010) y la más reciente Alianza del Pacífico (2011), en adelante la Alianza.

La Comunidad Andina de Naciones CAN, es un proyecto de los años 70's, actualmente participan de este organismo internacional Bolivia, Colombia, Ecuador y Perú. Por su parte, Chile y Venezuela fueron miembros, pero Chile se retiró bajo el régimen de Augusto Pinochet en 1976 y se reincorporó en calidad de miembro asociado el año 2006; mientras que Venezuela se retiró en el año 2006.

En la CAN funciona la Zona de Libre Comercio plenamente desde el año 2005, cuando Perú terminó su proceso de desgravación. En materia de movilidad de personas ha logrado que sea posible para los ciudadanos realizar actividades remuneradas en otro país de la Zona contando con una oferta o contrato de trabajo (basta con el registro como extranjero en el lugar de destino), mientras que para ingresar a los países para otros fines es suficiente con

${ }^{2}$ HerrerA, J C., «Las cláusulas de integración en las constituciones de Suramérica: 200 años después de la carta de Jamaica [*]», Colombia Internacional, n. ${ }^{\circ}$ 86, 2016, pp. 165-192

3 Acosta Alvarado, P. A., Más allá De La Utopía: Del Diálogo Interjudicial a La Constitucionalización Del Derecho Internacional. La Red Judicial Latinoamericana Como Prueba y Motor Del Constitucionalismo Multinivel, 2015. Tesis doctoral disponible en <http://eprints.sim.ucm.es/28119/> (última consulta, 15 junio 2016), p. 280. 
el documento de identidad nacional (sin visa e incluso sin pasaporte). Otros logros de la CAN son: la portabilidad de los aportes a seguridad social entre los cuatro países, la negociación en bloque de compra de medicamentos y un régimen común en materia de propiedad intelectual ${ }^{4}$.

La Asociación Latinoamericana de Integración $\mathrm{ALADI}^{5}$ es un proyecto que inició sus actividades en 1980. El propósito de este acuerdo es lograr un mercado común latinoamericano, actualmente es la asociación más grande de la región y cuenta con la participación de trece países. (Argentina, Bolivia, Brasil, Chile, Colombia, Cuba, Ecuador, México, Paraguay, Panamá, Perú, Uruguay y Venezuela).

Este acuerdo prevé la integración de manera progresiva, considerando la situación de cada país. En desarrollo de este postulado, por ejemplo, se han suscrito acuerdos que dan preferencias arancelarias sobre algunos productos a Bolivia, Ecuador y Paraguay sin reciprocidad ${ }^{6}$.

El Mercado Común del Sur MERCOSUR nace con el propósito de hacer contrapeso a los intereses de Estados Unidos sobre la región a principios de los años 90. La integración que propone si bien tiene un importante componente económico, también contiene compromisos sociales y políticos ${ }^{7}$. En esa medida, Mercosur no tiene una proyección de economía abierta, y más bien tiene un perfil casi proteccionista, sobre todo después de la redefinición política que ha sufrido desde el año $2003^{8}$.

La combinación de los elementos aperturistas y de compromisos sociales, hace que el bloque MERCOSUR sea clasificado como un híbrido entre los procesos de integración ya que no propone volver al proteccionismo, pero tampoco a la apertura total de sus mercados, sino que se encuentra en un punto intermedio, además, la presencia de compromisos en materia social y la preocupación por el crecimiento equitativo definen el perfil de este proceso de integración ${ }^{9}$.

${ }^{4}$ Comunidad Andina de Naciones. Quienes somos /logros; CAN, Decisión 486 del año 2000.

${ }^{5}$ LÓPEZ-JACOISTE DÍAZ, E., «Chapter 2. the Latin American Integration Association» en ODELLO, M.; and SEATZU, F. (eds)., Latin American and Caribbean International Institutional Law, Springer, 2015, pp. 22-42.

6 Aladi, Acuerdos Regionales - Apertura de Mercados (AR.AM) 1, 2 y 3.

7 Ochoa Bilbao, L.; Velázquez Flores, R., «México, el MERCOSUR y la alianza del pacífico: Intereses, identidades y prioridades regionales», Miríada: Investigación En Ciencias Sociales, 2016, vol. 7, n. ${ }^{\circ}$ 11, p. 53.

${ }^{8}$ Caballero Santos, S., «Mercosur: The Role of Ideas and a More Comprehensive Regionalism», Colombia Internacional, n. ${ }^{\circ}$ 78, 2013, pp. 127-144.

9 Briceño RuIZ, J., «Ejes y Modelos En La Etapa Actual De La Integración Económica Regional En América Latina», Estudios Internacionales (Santiago), vol. 45, n. ${ }^{\circ} 175$, 2013, p. 27. 
Participan de este proyecto Argentina, Brasil, Paraguay y Uruguay como fundadores; recientemente se unió Venezuela; y, actualmente, Bolivia está en proceso de adhesión.

Pese a la importancia económica de este bloque, solo ha firmado TLC con Israel, Egipto, Palestina y con la Comunidad Andina de Naciones. En días pasados, el gobierno del recién posesionado presidente de Argentina, Mauricio Macri, manifestó el interés de unirse a la Alianza del Pacífico en calidad de observador y a su vez, respecto de Mercosur, sostuvo que necesita un vínculo de salida hacia el pacífico ${ }^{10}$.

La historia de la Alianza Bolivariana para los Pueblos de Nuestra América ALBA, está íntimamente relacionada con el nacimiento de la Alianza del Pacífico y con el ocaso del proyecto estadounidense de Acuerdo de Libre Comercio de las Américas ALCA. Los países que hoy conforman la Alianza del Pacífico son justamente los países que estaban dispuestos a aceptar la suscripción del acuerdo con Estados Unidos, mientras que los países que lo rechazaron formaron el ALBA en 2004 ${ }^{11}$.

En noviembre del año 2005, la postura antagónica de los gobiernos de izquierda, encabezada por Hugo Chávez, rompió cualquier posibilidad de continuar con las negociaciones del ALCA, dejando claro que las posiciones políticas superaban las posibilidades de continuar con las negociaciones, además la formulación del nuevo acuerdo que daba vida al ALBA tenía claramente ingredientes de corte de reivindicación política y ánimos de consolidar el Socialismo del Siglo XXI. En palabras de BRICEÑO «el ALBA no es un forma tradicional de integración económica, sino que plantea que esta se desarrolle no a partir de una estrategia tradicional basada en el libre comercio, sino que (...) propone un nuevo modelo de integración económica no capitalista» ${ }^{12}$.

El ALBA estaba constituido en un principio por Argentina, Brasil y Venezuela, posteriormente se unieron Bolivia y Ecuador de Suramérica, y de Centroamérica: Antigua Barbuda, Cuba, Dominica, Granada, Nicaragua, San Cristóbal y Nieves, Santa Lucia, San Vicente y las Granadinas, y Surinam.

La Unión de Naciones Suramericanas UNASUR es una organización internacional que nace en el año 2004, tiene unos objetivos claramente políti-

${ }^{10}$ Diario La Nación. El gobierno instó al Mercosur a alinearse a la Alianza del Pacifico.

${ }^{11}$ Bernal-Meza, R., «ALIANZA DEL PACÍFICO Versus ALBA y MERCOSUR: Entre El Desafío De La Convergencia y El Riesgo De La Fragmentación De Sudamérica», Pesquisa \& Debate. Revista do Programa De Estudos Pós-Graduados Em Economia Política. vol. 26, n. ${ }^{\mathrm{O}} 1$ (47), 2015, p. 2.

${ }_{12}$ Briceño RuIz, J., «Ejes y Modelos En La Etapa Actual De La Integración Económica Regional En América Latina», Estudios Internacionales (Santiago), vol. 45, n. ${ }^{\circ} 175$, 2013, p. 18. 
cos en la región, sus alcances y pretensiones en materia económica son más bien limitados y centra su interés en la integración financiera y la cooperación económica y comercial, pero no tiene como objetivo crear zonas de libre comercio o crear un mercado común.

Sin embargo, la limitación respecto de la integración económica es una de las fortalezas de esta organización, ya que se centra en los aspectos sociales más problemáticos que conciernen a todos los países de la región. Y creo que es una fortaleza ya que permite darle continuidad a los programas independientemente de los perfiles políticos de cada periodo presidencial.

De este proyecto participan Argentina, Bolivia, Brasil, Chile, Colombia, Ecuador, Guyana, Paraguay, Perú, Surinam, Uruguay y Venezuela.

Y en último lugar, y de formación más reciente, está la Alianza del Pacífico, conformada por Chile, Colombia, México y Perú, caracterizada por su rápido crecimiento e impacto internacional y que corresponde al objeto de estudio este texto.

\section{CONTEXTO}

La Alianza del Pacífico nace el año 2010 como iniciativa del presidente peruano Alan García, los primeros pasos (primera cumbre) se dan en abril del 2011 con la participación de Colombia, Chile, Estados Unidos de México y Perú en calidad de miembros, también participó Panamá en calidad de observador y con la posibilidad de integrarse a la Alianza una vez adelante los Tratados de Libre Comercio con los demás países. El propósito de la Alianza es lograr la libre circulación de bienes, servicios, capital y personas dentro de la región. Por otra parte, una de las prioridades del proceso de integración es lograr una consolidación que favorezca el intercambio comercial con otros mercados, especialmente con los países de la cuenca del Pacífico asiático. Es notorio que la atención de la Alianza está centrada en la profundización de las relaciones económicas con un corte aperturista, dejando de lado el componente político y social que ha caracterizado a otras iniciativas de integración latinoamericanas.

Los cuatro países son democráticos y respetan el Estado de Derecho, otro aspecto relevante para su constitución es que sus políticas económicas son más o menos similares, son economías abiertas, los cuatro países tienen tratados de libre comercio con Estados Unidos y con la Unión Europea, a la cabeza está Chile con veintiún TLC y cinco Acuerdos Comerciales Preferenciales (AAP); seguido por México con trece TLC y seis APP; Perú por su parte quince TLC y una Unión Aduanera, y, por último, Colombia con ocho TLC, seis APP y una Unión Aduanera ${ }^{13}$.

13 Sistema de Información sobre Comercio Exterior SICE de la Organización de los Estados Americanos. Países: Chile, Colombia, México, Perú. 
En diciembre del año 2011 se celebró la segunda cumbre de la Alianza en Mérida, México. En esta cumbre se instruyó a los ministros de relaciones exteriores para que en un término inferior a seis meses concluyeran con el Acuerdo Marco que permitiera constituir el tratado fundacional de la Alianza. Además, se iniciaron las negociaciones sobre comercio electrónico e inversión, tratamiento arancelario, medidas sanitarias y fitosanitarias, y visa de la Alianza para facilitar la movilidad de personas.

La Alianza del Pacífico se funda sobre las bases formadas por los Tratados de Libre Comercio suscritos entre los países miembros, con la salvedad de que Colombia y Perú, por ser miembros de la CAN, tienen establecida un área de libre comercio y en esa medida no hacía falta suscribir un tratado adicional ${ }^{14}$.

Respecto del contexto geopolítico, algunas lecturas apuntan a que la Alianza nace como respuesta al crecimiento significativo de Brasil que lo posiciona como potencia y líder de la región; de ahí la importancia para los países miembros de aumentar su poder de influencia y participación en la región. Es más, esta visión, basada en el poder hegemónico, explica el interés y apoyo de Estados Unidos a la nueva formación ${ }^{15}$. Aunque esta motivación ha sido negada de manera categórica en diferentes oportunidades por los representantes de los Estados que conforman la Alianza, advirtiendo que se trata de una iniciativa abierta a la participación de otros Estados que compartan los mismos intereses ${ }^{16}$.

El potencial económico de la Alianza es innegable, el Pacífico es el océano más importante del mundo, cubre un tercio de la superficie terrestre, el $40 \%$ de la población de la tierra tiene acceso a éste (2.700 millones de personas) y concentra el 50\% del comercio del mundo, de ahí el interés de ampliar las relaciones comerciales a través de este océano.

En torno al Pacífico se han integrado tres bloques económicos adicionales a la Alianza del Pacífico ${ }^{17}$ : en primer lugar el Foro de Cooperación Económi-

${ }^{14}$ En el año 1994 se firmó TLC entre México y Colombia, en 1998 lo hizo Chile con México, en 2006 Perú con Chile y ese mismo año entre Chile y Colombia. Fuente: Sistema de Información sobre Comercio Exterior SICE. Mientras que Colombia y Perú, por ser parte de la CAN, rigen sus relaciones comerciales por la Zona de Libre Comercio que empezó a funcionar plenamente desde el año 2005. CAN Decisión 414 de 1997.

${ }_{15}$ Reggiardo Palacios, G., Un Nuevo Escenario Geopolítico En La Región Latinoamericana: La Creación De La Alianza Del Pacifico (2011-2015). Universidad Andina Simón Bolívar, Sede Ecuador, 2015.

${ }^{16}$ El Diario.es. La Alianza del Pacífico no es una guerra en Latinoamérica, asegura el presidente de Chile, Diario El Tiempo. 'Alianza del Pacífico no es un proceso contra nadie': Santos, Casa de América - Madrid. Presentación en España de la 'alianza del pacífico'.

17 Diario El Mercurio. A 500 años de su descubrimiento, el océano Pacífico concentra la mitad del comercio global. 
ca Asia Pacífico APEC, establecido en 1989 con el propósito de facilitar el intercambio comercial a través del Pacífico, este foro ha sido un espacio de entendimiento y cooperación, y si bien sus decisiones se toman por consenso y no son vinculantes, muestra la importancia y permanente interés en aumentar los lazos comerciales, el libre comercio y el desarrollo sostenible en torno a la la cuenca del Pacífico. De los países de la Alianza todos, a excepción de Colombia, forman parte de este foro.

En segundo lugar, el Acuerdo Transpacífico de Cooperación Económica TTP (Trans-Pacific Partnership) que es un tratado de libre comercio firmado por doce países en el año 2015 en el marco del APEC, del cual Colombia tampoco hace parte.

Y, en tercer lugar, la Asociación de Países del Sudeste Asiático ASEAN de 1967 y que desde el año 2007 está profundizando su integración.

Las economías de los países que conforman la Alianza se vieron fortalecidas por el aumento de los precios de los comodities durante su etapa de formación, mientras que se vivía una crisis con mayor dureza en Estados Unidos y la Unión Europea.

Las economías de los países individualmente consideradas son simplemente cuatro economías abiertas de tamaño pequeño a medio, sin embargo, unidas cuentan con una población de cerca de 215 millones de habitantes, con un PIB que corresponde al $37 \%$ de toda la región, además ocupa la novena posición entre las economías más grandes del mundo y en materia de negocios concentra el $50 \%$ del total de Latinoamérica ${ }^{18}$.

Los avances y logros de la Alianza se han visto favorecidos por la estabilidad y la unidad de perspectiva de los países miembros de la organización con respecto a la idea de desarrollo y crecimiento económico. De tal manera que las decisiones y la forma en que discurren las relaciones están determinadas no por una respuesta a una ideología o fervor patriótico sino por la certeza de que es el camino para lograr los objetivos de la organización y de cada país miembro.

Un ejemplo de esta visión se presentó con la llegada del presidente socialista Ollanta Humala al poder en el año 2011 que generó dudas con respecto al futuro del modelo de desarrollo económico de Perú. Dado el estado de inestabilidad política en otros países de la región, se temía que el nuevo gobierno se alineara al frente del Socialismo del Siglo XXI, tanto así que la bolsa experimentó una caída del $12 \%$ un día después de las elecciones y fue necesario que suspendiera sus operaciones ante el desplome; sin embargo,

${ }^{18}$ George, S., The Pacific Pumas. An Emerging Model for Emerging Markets.Washington: Bertelsmann Foundation y Global Economic Dynamics, 2014. Disponible en. $<$ http://www.bfna.org/publication/the-pacific-pumas-an-emerging-pacific-alliance-model-for-emerging-markets $>$ (última consulta, 15 junio 2016), p. 28. 
estas dudas fueron despejadas con los primeros meses de gobierno y se pudo constatar el compromiso del mandatario con los principios del Estado Social de Derecho y, sobre todo, con los principios democráticos. Es de anotar, que todos los avances de la Alianza se han adelantado bajo su gobierno, lo cual confirma la respuesta libre de ideología que ha fortalecido a la organización.

\section{CREACIÓN, ESTRUCTURA Y ORGANIZACIÓN}

El tratado constitutivo, denominado Acuerdo Marco de la Alianza del Pacífico, se suscribió el 6 de junio de 2012 en Paranal, Chile. Este acuerdo define como requisito esencial para participar del proyecto la vigencia del Estado de Derecho, la democracia y el orden constitucional; también, la separación de los poderes del Estado y la protección, promoción y respeto de los derechos humanos y libertades fundamentales ${ }^{19}$.

\subsection{Objetivos}

Los objetivos que se ha fijado la Alianza, son fundamentalmente de carácter económico, así pues, establecer un área de integración con miras a lograr la libre circulación de bienes, servicios, capitales y personas; asimismo, impulsar el crecimiento económico y por último convertirse en una "plataforma de articulación política, de integración económica y comercial, y de proyección al mundo, con especial énfasis al Asia Pacífico» ${ }^{20}$.

De tal suerte, que la Alianza del Pacífico pretende crear un mercado común en un momento dado, solo de esta manera pueden ser interpretados estos objetivos que se ha trazado ${ }^{21}$.

Para alcanzar estos objetivos se pretende liberalizar el intercambio comercial de bienes y servicios, creando así una zona de libre comercio; además, establecer la libre circulación de capitales y promover la inversión entre la partes; también, se busca promover la colaboración entre las autoridades migratorias y consulares con el propósito de facilitar el movimiento de personas en el interior de la zona y contribuir en el desarrollo de mecanismos para la contención de la delincuencia organizada; e impulsar la Plataforma de Cooperación del Pacífico.

Estos objetivos confirman la renuencia a lograr una integración con un componente social o político, y en vez de eso, lograr un impacto significa-

19 Alianza del Pacífico, «Acuerdo Marco de la Alianza del Pacífico» Art. 2.

20 Ibid. Art. 3.

${ }^{21}$ Briceño RuIz, J., «Ejes y Modelos En La Etapa Actual De La Integración Económica Regional En América Latina», Estudios Internacionales (Santiago), vol. 45, n. ${ }^{\circ} 175$, 2013, p. 30. 
tivo a través de decisiones pragmáticas que den fluidez a las relaciones económicas ${ }^{22}$.

El artículo 9 del Acuerdo Marco dispone que: «La Alianza del Pacífico promoverá iniciativas y lineamientos de acción sobre temas de interés regional o internacional y buscará consolidar mecanismos de vinculación con Estados y organizaciones internacionales». Adicionalmente, dispone que las organizaciones internacionales pueden apoyar a la nueva formación en el logro de sus objetivos.

\section{2. Órganos y funcionamiento}

\subsubsection{Cumbres}

Los encuentros de los presidentes de los Estados miembros y de los observadores invitados se denominan Cumbres, en ellas se marca la senda, los objetivos y la agenda por donde debe continuar el proceso de integración. El resultado de las cumbres es recogido en las Declaraciones, donde se exponen las conclusiones, lineamientos y propósitos para continuar con el proceso de integración. Estos encuentros tienen un valor diplomático y político importante aunque no forman parte de la estructura de toma de decisiones de la Alianza del Pacífico, por esa razón, las Declaraciones son manifestaciones de intenciones no vinculantes.

\subsubsection{Presidencia pro tempore}

La Alianza no cuenta con una secretaría permanente y por eso está previsto que la coordinación de las actividades de la organización sean alternadas por los países que la conforman bajo la figura de la presidencia pro tempore.

El Acuerdo Marco, en su artículo 7, establece que es responsabilidad del titular de la presidencia organizar las cumbres, coordinar las reuniones del Consejo de Ministros y del Grupo de Alto Nivel (GAN), llevar el registro y actas de los encuentros, y representar a la Alianza en los asuntos de interés común por encargo de las partes.

Es evidente pues, que la presidencia tiene un contenido administrativo y relativamente de poca importancia respecto del desarrollo del proceso de integración, ya que no tiene poder de decisión e incluso la representación se ve condicionada al «encargo de las Partes».

22 OchoA Bilbao, L.; VelázQuez Flores, R., «México, el MERCOSUR y la alianza del pacífico: Intereses, identidades y prioridades regionales», Miriada: Investigación En Ciencias Sociales, 2016, vol. 7, n. ${ }^{\circ}$ 11, p. 59. 


\subsubsection{El Consejo de Ministros}

El artículo 4 del Acuerdo Marco establece que el Consejo de Ministros estará integrado por los Ministros de Relaciones Exteriores y de Comercio Exterior o sus delegados de los países miembros.

El Consejo de Ministros es el espacio de decisión más importante de la Alianza y es el encargado de tomar las medidas más relevantes para desarrollar y cumplir con las disposiciones y objetivos del Acuerdo Marco y de las Declaraciones presidenciales.

Además de tomar la decisiones, tiene la función de supervisar y evaluar la ejecución de las mismas; también, establece la agenda, crea los grupos de trabajo encargados de profundizar en la consecución de los fines de la Alianza, define la política de la Alianza en relación con otros Estados o esquemas de integración y puede convocar al Grupo de Alto Nivel GAN cuando lo considere necesario. El Consejo de Ministros se reúne una vez por año y sesiona con la presencia de todas las partes, sin embargo puede reunirse extraordinariamente a solicitud de cualquiera de las partes.

Las decisiones tomadas por el Consejo son producto del consenso y se consideran parte del ordenamiento jurídico de la Alianza (Art. 6 del Acuerdo Marco). Sin embargo, hay que aclarar que las decisiones adoptadas por el Consejo, no modifican ni reemplazan otros acuerdos comerciales que las partes hayan suscrito entre ellas, ya sean regionales, multilaterales o bilaterales.

\subsubsection{El GAN}

El Grupo de Alto Nivel está conformado por los viceministros de relaciones exteriores y de finanzas de los Estados parte, este grupo es el encargado de supervisar los avances de los grupos técnicos y comités.

\subsubsection{Comités y Grupos Técnicos}

Actualmente la Alianza cuenta con una veintena de comités ${ }^{23}$, los cuales tienen diferentes tareas, que van desde estudiar situaciones concretas, hasta formular propuestas para avanzar en el proceso o simplemente dar a conocer o promocionar la Alianza y sus ventajas. Es evidente que todos los comités,

${ }^{23}$ Entidades de promoción, Asuntos Institucionales, Comercio e Integración, Comité de Expertos CEAP (Consejo Empresarial de la Alianza del Pacífico), Compras Públicas, Cooperación, Desarrollo Minero, Educación, Estrategia Comunicación, Innovación, Mejora Regulatoria, Movilidad de Personas, Propiedad Intelectual, PYMES, Relacionamientos Externo, Servicios y Capitales, Transparencia Fiscal, Turismo, Género, Cultura. 
a excepción de los de Género $^{24}$ y Educación, están diseñados para causar impacto económico; incluso, el comité de Cultura tiene como objetivo «impulsar el desarrollo de los emprendimientos de las industrias culturales y creativas de los países miembros, aprovechando su alto potencial productivo y dinamizador de una economía sostenible que, al mismo tiempo, construya y proyecte internacionalmente la identidad y diversidad de la Alianza del Pacífico» ${ }^{25}$.

\subsubsection{Países observadores}

Para los países que no son miembros, es posible vincularse a la Alianza en calidad de país observador, para ello el Consejo de Ministros debe aprobar la participación del Estado por unanimidad y con la aprobación se definirán las condiciones de dicha participación. La Alianza está abierta a la adhesión de nuevos miembros con la condición de que dicho Estado tenga acuerdos de libre comercio con cada una de las partes que la conforman ${ }^{26}$.

\subsubsection{Consejo Empresarial de la Alianza del Pacífico}

También se ha instaurado el Consejo Empresarial de la Alianza del Pacífico CEAP cuyo propósito es promover la Alianza y elevar sugerencias y recomendaciones para mejorar el proceso de integración. Es de indicar que el CEAP está constituido por cuatro capítulos, uno por país, y cada capítulo está integrado por cuatro miembros «hombres de negocios reconocidos». El objetivo es que el sector privado participe activamente en la formación de la Alianza, creando un canal de comunicación directo con los empresarios. Este Consejo no hace parte de la estructura orgánica prevista en el Acuerdo Marco de la Alianza, y su constitución fue suscrita por cuatro empresarios designados por los presidentes de cada país.

\section{AVANCES Y LOGROS}

Junto con la suscripción del Acuerdo Marco, se dio paso la Declaración de Paraná del 6 de Junio del año 2012, en ella se celebró la finalización de la negociación en materia de comercio electrónico y se impulsaron nuevos objetivos como la eliminación de aranceles, la formulación de un régimen de solución de controversias, implementación de la certificación electrónica de

${ }^{24}$ La descripción sobre este comité solo dice que es un elemento transversal de la Alianza del Pacífico. Temas de Trabajo/cultura.

25 Ibid.

26 Alianza del Pacífico, Acuerdo Marco de la Alianza del Pacífico. Art. 11. 
origen, cooperación en materia aduanera, reconocimiento de Operador Económico Autorizado, avance en la regulación de medidas sanitarias y fitosanitarias; además, se promovió la creación de una plataforma de movilidad estudiantil, en todos los grados, para que desde el año 2013 empezase a funcionar; del mismo modo, se promovió la realización de eventos culturales que reflejen el patrimonio cultural común. En ese mismo sentido, se fijaron como objetivos inmediatos la identificación de obstáculos a la inversión y el comercio en los países miembros con el fin de eliminarlos; la supresión de visas (por parte de México especialmente); el impulso de la cooperación en materia de seguridad para facilitar la movilidad, y, por último, se ordenó a las agencias de comercio internacional de los países miembros buscar inversiones y profundizar la exploración de nuevas regiones para instalar representantes de promoción de dichas agencias, con especial énfasis en Asia.

La Alianza ha establecido herramientas comerciales para consolidarse como un instrumento de desarrollo de la economía regional, sin embargo, también ha implementado medidas y proyectos no comerciarles para impulsar y profundizar el proceso de integración, así pues, la Red de Investigación Científica sobre Cambio Climático, la creación de la Plataforma Estudiantil, o la constitución del Fondo de Cooperación de la Alianza del Pacífico, son medidas que coadyuvan al proceso de integración pero sin una naturaleza comercial. A continuación se presentan los principales logros y avances de la Alianza.

La creación de una Zona de Libre Comercio es sin lugar a dudas el avance más importante en la consolidación de la Alianza. El Protocolo Adicional que la crea fue suscrito el día 10 de febrero del año 2014 en Cartagena de Indias y entró en vigencia el 1 de mayo de 2016 luego de ser aprobado mediante los procedimientos internos de cada país. Este acuerdo libera de aranceles el $92 \%$ de los productos y se prevé que el $8 \%$ restante se eliminen en el corto y mediano plazo; sin embargo, queda un bajo porcentaje sobre productos «altamente sensibles» diferido a 17 años. El objetivo es que para el año 2030 todos los productos circulen libremente ${ }^{27}$.

Con la entrada en vigencia de este Protocolo adicional, también se pone en marcha el Procedimiento de Solución de Controversias, el cual está dispuesto con el fin de resolver las disputas entre un inversionista de un Estado parte y un Estado parte (Capítulo 10 sobre inversiones, Sección B); y también el Procedimiento de Solución de Diferencias, que se convierte en el marco para resolver los posibles conflictos entre las partes del Acuerdo $(\mathrm{Ca}-$ pítulo 17).

27 Alianza del Pacífico, Dirección general de comunicación social. Comunicado conjunto de los países miembros de la AP con motivo de la entrada en vigor del protocolo adicional al acuerdo marco. 
Los tratados previos suscritos entre los países continúan vigentes en los temas no regulados por el tratado de la Alianza. La ventaja que aporta este tratado es la armonización de las normas para los cuatro países lo cual es un paso hacia adelante para mejorar y facilitar las relaciones comerciales.

Uno de los cambios más importantes en materia de libre comercio es el avance en materia de Reglas de Origen, ya que todos los productos provenientes de los países de la Alianza estarán cubiertos por la Certificación de Origen. Esta medida, facilita la creación de cadenas productivas eficientes y competitivas, lo cual se traduce en que para un empresario sería posible comprar materia prima en Perú y Chile, fabricar en Colombia y vender a México manteniendo las preferencias de producto de origen, es más, ese producto puede venderse a Estados Unidos (sin aranceles), en virtud del TLC que todos los países tiene suscrito con ese país.

También se avanzó en materia de medidas sanitarias y fitosanitarias, eliminando la posibilidad de que haya un trato discriminatorio o que éstas se conviertan en barreras de acceso al mercado común.

Respecto de la contratación pública se ha dado lugar a la apertura, el artículo 8 del protocolo prevé que todos los proveedores de los países de la Alianza deben ser tratados tan favorablemente como a los nacionales, se acordaron medidas para garantizar la transparencia y la publicidad de los procedimientos de contratación.

Otro aspecto que ha permitido la profundización del libre mercado es la inclusión de las telecomunicaciones en el Protocolo Adicional. Este tema no tenía desarrollo en los acuerdos bilaterales firmados por Chile con Colombia y Perú28. Esto demuestra que si bien la Alianza se apoya firmemente en los acuerdos ya firmados, no se limita éstos e indica la pretensión y determinación de las partes en ampliar los ámbitos de aplicación del libre comercio.

El Mercado Integrado Latinoamericano MILA es la integración de las bolsas de valores de los cuatro países, suele ser presentado como un avance de la Alianza del Pacífico, sin embargo, es importante aclarar que la integración de las bolsas de valores de Bogotá, Lima y Santiago de Chile inició desde el año 2009 y entró a operar desde el 30 de mayo de 2011, es decir, este proceso es independiente de la Alianza, por lo menos en un principio ${ }^{29}$. Lo que sí es un logro, es la integración de la bolsa de valores de México en el año 2014. Además, hoy el MILA se presenta como "somos el Mercado de Valores de la Alianza del Pacífico-Cuatro países un Mercado» ${ }^{30}$.

Actualmente, el propósito de la integración lograda con el MILA es facilitar el acceso al mercado de inversión de renta variable de todas las bolsas

\footnotetext{
28 Alianza del Pacífico. Protocolo Adicional al Acuerdo Marco ¿Qué significa?.

${ }^{29}$ Mercado Integrado Latinoamericano MILA. Reseña histórica.

${ }^{30}$ Ibid.
} 
con la mera participación de un intermediario local, el cual puede llevar a cabo las operaciones en cualquiera de las cuatro bolsas integradas. También están integrados los depósitos de valores de los cuatro países.

Este tipo de integración es novedosa en la región y posiciona al mercado en el primer lugar de empresas listadas y de operaciones. Respecto de las condiciones, cada bolsa conserva su autonomía y las operaciones se realizan en moneda local. Esto es debido a que la integración no se realizó a través de la fusión de las bolsas, sino mediante la adecuación de medios tecnológicos que permitieran el funcionamiento integrado de las cuatro bolsas. Dado que no hay una fusión, cada bolsa conserva su independencia en materia de regulación, control y vigilancia de la actividad ${ }^{31}$.

Pese a que hoy el alcance del MILA es limitado (inversiones de renta variable), en la Declaración de Paracas de 3 de julio de 2015, producto de la X Cumbre de la Alianza del Pacífico, se ordenó a los Ministros de Finanzas de la Alianza que identifiquen y coordinen iniciativas que permitan el fortalecimiento y la consolidación del MILA, igualmente, que se implementen medidas que logren el reconocimiento de emisores de ofertas públicas primarias, y se amplíe la cantidad de instrumentos negociables dentro del mercado. También se instruyó a los ministros para avanzar en la homologación de tratamiento fiscal y evitar la doble imposición.

Resulta claro que el MILA avanza con el mismo modelo de integración de la Alianza del Pacífico en el sentido de lograr acuerdos concretos con efectos inmediatos y palpables que se consolidan y dan lugar a que resulten eficientes y atractivos para el ámbito internacional.

El Acuerdo sobre medicamentos, también significa un avance para la Alianza, este acuerdo fue firmado por los directores de las autoridades sanitarias de los cuatro países y busca facilitar «el intercambio de información relacionada con la evaluación del otorgamiento de registro sanitario», es decir, que las autoridades van a aprovechar la información obtenida por las otras agencias para agilizar los procesos, sin embargo, y ante la ausencia de normas comunes o armonizadas, cada autoridad conserva su discrecionalidad y deberá aplicar las normas vigentes en cada país. Hasta este momento no hay homologación de certificados. Así pues, la certificación de Buenas Prácticas de Manufactura (BPM), posiblemente sí se verá agilizada ya que puede basarse en las actas e información obtenida por otra agencia que sea parte del acuerdo $^{32}$.

31 ARIZA VILlar, D. M.; Díaz Moya, M. F., Mercado Integrado Latinoamericano (MILA): ¿ Objetivos no Cumplidos?, Repositorio Institucional de la Universidad EAFIT, 2015, pp. 9 y 10.

32 Alianza del Pacífico. Acuerdo Interinstitucional de Cooperación que Celebran las autoridades sanitarias de la Alianza del Pacífico. 20 de Junio de 2013. 
Por otra parte, el desarrollo de la actividad diplomática y comercial conjunta ha llamado la atención y se ha mostrado como un éxito de la Alianza. En virtud de este acuerdo, los países pueden incrementar su representación y presencia diplomática a través de embajadas y consulados conjuntos. Además, el acuerdo prevé el compromiso de prestar atención a los ciudadanos de otros países miembros de la Alianza, en caso de emergencias y cuando el país que solicita la cooperación no tenga presencia diplomática alli ${ }^{33}$.

En desarrollo de este acuerdo se han establecido varias embajadas conjuntas, por ejemplo la de Colombia y Perú en Vietnam; en Ghana los cuatro países comparten la sede diplomática; también en Singapur funcionan conjuntamente la de México y Colombia, entre otras. Incluso, España se ha mostrado interesada en unirse a este proyecto de representación diplomática conjunta dadas las posibilidades de ampliación de la cobertura que supone ${ }^{34}$. Por otra parte, se dio lugar a la apertura de dos agencias de promoción de la Alianza, una en Estambul (Turquía) y otra en Casablanca (Marruecos).

Otra medida no comercial es la creación de la Plataforma Estudiantil de la Alianza del Pacífico, consistente en un programa de becas de intercambio estudiantil y docente; hasta este momento ha logrado la movilización de casi mil participantes desde su entrada en funcionamiento, una particularidad es que el programa no cuenta con una apropiación de recursos para llevarla a cabo, sino que se basa en el compromiso mutuo entre diferentes entidades ${ }^{35}$ de los países de otorgarlas de manera recíproca, en tal sentido, las convocatorias y postulaciones se hacen directamente con cada país y no en una institución que las centralice; cada país se compromete a otorgar cien becas por año ( 75 para estudios de grado y las 25 restantes para doctorados y movilidad de profesores).

La Red de Investigación Científica en materia de Cambio Climático es una de la iniciativas que ya ha tenido repercusión internacional debido a que los resultados de su primera investigación fueron presentados de manera conjunta en la Conferencia de las Partes sobre Cambio Climático de la ONU en Lima de $2014 \mathrm{COP} 20^{36}$, dando lugar a una declaración que recoge los compromisos de los cuatro países sobre la materia ${ }^{37}$. Estos son gestos que

${ }^{33}$ Las relaciones entre Colombia y Perú también se ven determinadas por la CAN, por ejemplo, en materia de migración laboral, estos dos Estados ya tienen una regulación Decisión 545, que otorga preferencias en esta materia, que incluso son más ambiciosas que las acordadas con la Alianza el Pacífico.

${ }^{34}$ Diario ABC.ES, España quiere compartir embajadas con los países de la Alianza del pacifico.

35 AGCID, Chile; ICETEX, Colombia; AMEXCID, México; PRONABEC, Perú.

36 Alianza del Pacífico. Temas de Trabajo/Cooperación.

37 Ministerio de Relaciones Exteriores de Colombia. Alianza del Pacífico presentó declaración en materia de cambio climático en la conferencia de las partes sobre cambio 
proyectan a la Alianza del Pacífico como un proceso de integración sólido. La red está formada por representantes de los Ministerios de Medio Ambiente entre otras instituciones de cada país. Después del reconocimiento de ese primer trabajo, en la Declaración de Paracas se encargó la continuación de otro proyecto con el apoyo de la Universidad de Oxford en materia de Biodiversidad.

Se estableció la Plataforma de Cooperación del Pacífico a través de un memorándum de entendimiento firmado por los ministros de relaciones exteriores con el propósito de apoyar actividades de cooperación en materia de medio ambiente y cambio climático; innovación, ciencia y tecnología; micro, pequeña y medianas empresas PYMES; y desarrollo social.

En el acuerdo se fijan, entre otros aspectos, los requisitos mínimos que deben tener los proyectos de cooperación, tales como los objetivos, el cronograma y los responsables del mismo.

En ese mismo sentido, se ha suscrito el Acuerdo para el establecimiento del Fondo de Cooperación de la Alianza del Pacífico, esté sí con rango de tratado, que tiene por objeto «financiar programas, proyectos y actividades de cooperación aprobados por el Grupo Técnico de Cooperación de la Plataforma de Cooperación del Pacífico (GTC)». Este acuerdo fija un aporte de US \$250.000 Dólares americanos por parte de cada miembro ${ }^{38}$.

También se ha firmado un Acuerdo de Cooperación en materia de turismo, en el Marco de la Plataforma de Cooperación, que tiene por objeto potenciar las posibilidades que ofrece la región en esta área. El Acuerdo tiene como fin la promoción y divulgación del sector especialmente en «turismo de aventura y naturaleza; turismo deportivo, turismo de sol y playa, turismo de cultura y turismo organizado para grupos; turismo de eventos y convenciones». Este acuerdo se ha traducido en la participación conjunta en macro ruedas de negocios, ferias internacionales, y exposiciones. Es de destacar, que el Acuerdo, de manera explícita, establece que no genera obligaciones jurídicamente vinculantes para las partes.

En el año 2013 se constituyó la Comisión Interparlamentaria de la AP, en primer lugar en el mes de febrero se reunieron en Lima los presidentes de los cuatro parlamentos de los países y se acordó, mediante una Declaración conjunta, adelantar los procedimientos internos para la aprobación de Acuerdo Marco que daría vida a la Alianza. Posteriormente, en mayo del mismo año, en Bogotá, se firmó el acuerdo por parte de los presidentes de los parlamen-

climático de la $O N U$.

${ }^{38}$ Corte Constitucional del Colombia. Sentencia C-106/16. La ley aprobatoria de este tratado fue declarada inexequible por la Corte Constitucional por vicios de trámite en su formación (falta de verificación del quórum). Esto obliga a que el procedimiento deba repetirse. 
tos mediante el cual se formalizaba la Comisión de Seguimiento Parlamentario al Acuerdo de la Alianza del Pacífico ${ }^{39}$.

Cada parlamento se compromete a formar una comisión de por los menos seis miembros, quienes a su vez integrarán el pleno de la Comisión de Seguimiento del Acuerdo de la Alianza Pacífico. Al momento de escribir estas líneas se está discutiendo, por parte de Perú y Chile, unificar el marco normativo y los procedimientos para hacer el seguimiento del acuerdo ${ }^{40}$.

Sobre movilidad de personas los avances son discretos pero significativos. El más importante fue la supresión de visados por parte de México para los ciudadanos de Colombia y Perú. El panorama general es la libertad de movimiento de turistas y personas de negocios, que quedan autorizados para ingresar y permanecer en el país anfitrión por aproximadamente 180 días al año sin necesidad de visa; en todo caso no se prevé que bajo esta modalidad se puedan desarrollar actividades remuneradas permanentes.

Con el fin de profundizar las relaciones, se creó el Programa de Vacaciones y Trabajo, que es un visado que se expide a los ciudadanos jóvenes (entre 18 y 30 años) de los países de la Alianza. Este visado permite desarrollar actividades remuneradas por medio tiempo, durante un periodo determinado (depende del país) y por máximo 12 meses, con la condición de que la actividad principal del interesado no sea el trabajo. Este avance se logró gracias al «Acuerdo Interinstitucional de la Alianza del Pacífico para un Programa de Vacaciones y Trabajo» firmado por ministros de relaciones exteriores en el año 2014.

La Alianza cuenta con la participación de cuatro países suscriptores que suman 216 millones de habitantes, un PIB de 2,1 Billones de Dólares (38\% del PIB regional); tasa de inflación promedio del 3,9\%, y la de desempleo asciende a un 6,5\%; también cuenta con la participación de cuarenta países observadores, de los cuales dos (Costa Rica y Panamá) son candidatos a entrar a formar parte en calidad de miembros ${ }^{41}$.

La Alianza se ha caracterizado por tener objetivos ambiciosos, así lo han dejado ver las diferentes Declaraciones y lo más importante es que el contenido de éstas se ha visto desarrollado en acuerdos y medidas que demuestran avances respecto del cumplimiento de la agenda.

${ }^{39}$ El nombre utilizado es Comisión Interparlamentaria de la Alianza del Pacífico, sin embargo, en el Acuerdo se denominó Comisión de Seguimiento Parlamentario al Acuerdo de la Alianza del Pacífico y en los medios de comunicación suele utilizarse Comisión Interparlamentaria de la Alianza del Pacífico.

40 Oficina Regional para América Latina y el Caribe. Notas Sobre Políticas Para La Formalización De Las Micro y Pequeñas Empresas. Organización Internacional del Trabajo, 2014. Disponible en <http://www.ilo.org/wcmsp5/groups/public/---americas/--ro-lima/documents/publication/wcms_318203.pdf $>$ (última consulta, 15 junio 2016).

${ }^{41}$ Alianza del Pacífico. Abecé de la Alianza del Pacífico. 


\section{PERSPECTIVAS}

Una ventaja significativa de la Alianza Pacífico es su capacidad para amortiguar los efectos del modelo «hub and spoke» propios de los TLC con Estados Unidos ${ }^{42}$, permitiendo que la integración entre las economías de los países parte asuman un intercambio directo entre ellas y evitando la necesidad de pasar por el «hub» (Estados Unidos). Esto quiere decir, que el papel de intermediario ante el aislamiento de las economías latinoamericanas previo a la integración se ve disminuido, facilitando los flujos comerciales, potenciando los intercambios multilaterales y superando los meramente bilaterales.

En ese mismo sentido, la Alianza del Pacífico reanuda las actividades de apertura económica de carácter regional de los años 90's, superando las iniciativas que basan su idea de crecimiento en la mera integración regional interna ${ }^{43}$. De ahí el interés de explorar nuevas posibilidades de mercado, especialmente en Asia.

La Alianza no ha creado una institucionalidad con personalidad jurídica propia y en esa medida no es posible escindir la voluntad de los Estados de la voluntad de la Alianza ${ }^{44}$. Y hasta este momento no parece que haya intención de crear una organización que sea sujeto de derecho internacional. En ese orden de ideas, la Alianza no tiene capacidad para suscribir tratados ni hacerse parte frente a los tribunales que solo admiten Estados.

«De suyo, al no comportar la naturaleza jurídica de una organización interestatal, la Alianza del Pacífico no podrá adoptar, convenir ni suscribir instrumentos internacionales gobernados por el derecho internacional público. Tampoco será procedente la asignación de prerrogativas o privilegios, ni el reconocimiento de inmunidades, a manera de régimen general internacional, en la medida en que la Alianza del Pacífico no es, jurídicamente, diferenciable de los Estados que la constituyeron y, por lo tanto, carece de autonomía respecto de ellos. Ello, naturalmente, sin perjuicio de que tales Estados continúen disfrutando, de manera independiente, de su régimen de privilegios e inmunidades, en calidad de sujetos de derecho internacional independientes $\rangle^{45}$.

42 Tole, J. J., «Los TLC De Estados Unidos Con Países Latinoamericanos: Un Modelo De Integración Económica "Superficial" Para El Continente Americano», Revista Derecho Del Estado, vol. 30, n. ${ }^{\circ}$ 30, 2013, pp. 251-300.

${ }^{43}$ Gonzalez-Perez, M. A., (et al)., «Pacific Alliance: Bringing Back Open Regionalism to Latin America», México y La Cuenca Del Pacífico, vol. 4, n. ${ }^{\circ} 11,2015$, pp. 21-51.

${ }^{44}$ Díaz-Cediel, S., «La Alianza Del Pacífico: Un Escrutinio Jurídico Al Tenor Del Derecho De Las Organizaciones Internacionales», ACDI-Anuario Colombiano De Derecho Internacional, vol. 9, 2016, p. 380.

${ }^{45}$ Ibid. p. 381. 
Los países participantes decidieron conservar la soberanía y adelantar el proceso de integración manteniendo la posibilidad de negociar todos y cada uno de los aspectos de la Alianza evitando crear una estructura externa como espacio de decisión. De tal manera que se creó un Acuerdo Marco genérico, con unos objetivos claros pero libres de compromisos específicos que llegaran a traducirse en obligaciones. Los pasos para profundizar la integración, quedan condicionados a la negociación individual de cada uno de los temas.

Esta característica puede suponer una limitación respecto del desenvolvimiento de las relaciones de la Alianza con otros Estados o sujetos de derechos internacional, especialmente a la hora de concretar los acuerdos. No obstante, el hecho de que las decisiones que se tomen en el marco de la Alianza necesiten del consenso de los Estados les difiere mayor legitimidad, ya que los escenarios donde la voluntad del Estado y la voluntad de la organización sean contrarias son virtualmente inexistentes.

El hecho de que la Alianza no se haya constituido con los elementos propios de una organización internacional, con personalidad jurídica propia, puede ser la razón del dinamismo que ha demostrado en la toma de decisiones y que le ha permitido despertar el interés de la comunidad internacional ${ }^{46}$.

La Alianza presenta otra particularidad esencial y es que su modelo de integración está basada en el TLCAN (Tratado de Libre de Comercio América del Norte) que es el paradigma del regionalismo estratégico norte-sur, es decir, entre países con asimetrías (desarrollados y en vías de de desarrollo). Pues bien, esto hace que el proyecto de la Alianza, pese a ser un acuerdo sursur (entre países en vías de desarrollo), se presente como una novedad dentro de los procesos de integración. Sin embargo, es importante tener en cuenta que todos los países que conforman la Alianza tienen suscritos TLC con Estados Unidos, lo cual hace que en sus ordenamientos estén vigentes normas de tipo OMC plus, y en esa medida se trata de lograr una convergencia de esta normativa ${ }^{47}$.

La Alianza ha sido vista como una consolidación de los intereses de los Estados Unidos dentro de la región, convirtiéndose en un desafío político e incluso diplomático que puede comprometer las relaciones con los demás países de la región.

La integración de economías diversificadas con economías rezagadas es otra característica de esta unión, Chile tiene una economía abierta y altamente diversificada, mientras que la de Colombia está mucho más atrasada en este aspecto. Con todo, la mera integración económica no se traduce en cre-

${ }^{46}$ Ibid. p. 385.

${ }^{47}$ Briceño RuIz, J., «Ejes y Modelos En La Etapa Actual De La Integración Económica Regional En América Latina», Estudios Internacionales (Santiago), vol. 45, n. ${ }^{\circ} 175$, 2013, pp. 31 y ss. 
cimiento de la economía ni en aumento de la productividad, tampoco conlleva una disminución de la informalidad que aqueja a los países de la región ${ }^{48}$, en ese sentido, es indispensable que estos aspectos entren a formar parte de la agenda de la Alianza.

\section{CONCLUSIONES}

La Alianza del Pacífico es un proyecto responde a un conjunto de intereses compartidos por los cuatro países que han decidido participar de él, es una apuesta por superar el regionalismo latinoamericano impregnado por la ideología que ha marcado los últimos años de procesos de integración y en su lugar aprovechar las oportunidades que ofrece la apertura a nuevos mercados.

Si bien los objetivos son ambiciosos y se han puesto en marcha proyectos importantes como la movilidad de estudiantes y profesores, o la constitución de un fondo para crecer la infraestructura los países, la Alianza se queda corta en proyectos que despierten el interés y construyan una idea de la Alianza que resulte importante y significativa para los ciudadanos.

No es posible desconocer las críticas al modelo de desarrollo basado en la apertura económica, sin embargo, la Alianza, a mi modo de ver, es una forma de sacar provecho del marco ya existente ${ }^{49}$, y en esa medida considero que es ventajoso para las economías de los países emergentes buscar espacio en diferentes mercados y, sobre todo, la posibilidad de negociar en bloque.

El modelo que ha implementado la Alianza es novedoso ya que la integración no está basada en la creación de una institucionalidad nueva con carácter internacional, sino que echa sus raíces en los tratados ya existentes entre los países miembros y entre éstos y otros mercados.

Los avances que logra (o ha logrado) son vertidos en nuevos tratados independientes en ausencia de instancias supranacionales; esta característica brinda dinamismo en la toma de decisiones y garantiza mayor autonomía a los países parte en las negociaciones; esto es especialmente notorio si se

${ }^{48}$ Los países que conforman la Alianza han tomado algunas medidas para disminuir la informalidad en jóvenes. Vide: OFICINA REGIONAL PARA AMÉRICA LATINA Y EL CARIBE. Notas Sobre Políticas Para La Formalización De Las Micro y Pequeñas Empresas. Organización Internacional del Trabajo, 2014. Disponible en $<$ http://www.ilo. org/wcmsp5/groups/public/---americas/---ro-lima/documents/publication/wcms_318203. pdf $>$ (última consulta, 15 junio 2016); OFICINA REGIONAL PARA AMÉRICA LATINA Y EL CARIBE, Formalizando La Informalidad Juvenil: Experiencias Innovadoras En América Latina y El Caribe Organización Internacional del Trabajo, 2015. Disponible en $<$ http://www.ilo.org/wcmsp5/groups/public/---americas/---ro-lima/documents/publication/wcms_359270.pdf> (última consulta, 15 junio 2016).

49 Todos los países tienen firmados tratados de libre comercio entre ellos, con Estados Unidos y con la Unión Europea. 
compara con otras iniciativas que han optado por estructuras burocratizadas. Todo lo anterior, se traduce en flexibilidad respecto de la agenda, el contenido y la profundidad de los acuerdos. Pero esta flexibilidad también trae como desventaja que las posibles diferencias entre los países se tornen en temas vetados que impidan la continuación y profundización de la iniciativa.

Otro aspecto que hace que la Alianza sea relativamente frágil es que muchos de sus avances están basados en acuerdos interinstitucionales (entre autoridades de cada país), y en este sentido, la falta de una institucionalidad (una propia de la Alianza) que lo soporte se hace evidente, muchos de los avances que se se anuncian como propios de la Alianza dependen de estos acuerdos de menor jerarquía, y en esa medida necesitan de la voluntad de las partes e incluso de los gerentes o directores de las unidades administrativas de cada país; esto no significa un problema mientras las relaciones sean fluidas y no haya asperezas, sin embargo, que las estructuras que dan vida a los avances estén desprotegidas es un hecho que no puede pasar desapercibido.

También es de destacar la importancia del consenso en la toma de decisiones, lo cual ha significado la proyección de una imagen de unidad y determinación para continuar con el proceso.

Es posible que los mecanismos adoptados hasta ahora resulten suficientes para dar un primer impulso al proceso. Sin embargo, si la agenda y los objetivos aumentan, y si la ambición real es lograr un mercado común y no solo un Área de Libre Comercio, estos mecanismos se revelarán insuficientes y será indispensable la creación de una estructura supranacional que blinde los procesos y avances logrados hasta hoy y que formule los futuros. Esto significaría una nueva etapa en el proceso de integración, con unas nuevas dinámicas donde tomaría especial relevancia el ejercicio democrático y la construcción de ciudadanía latinoamericana.

Una palabra íntimamente ligada a toda la literatura acerca de la Alianza del Pacífico es «potencial», desde la prensa, pasando por la información oficial, hasta llegar a los círculos académicos; la razón que encuentro para que esto sea así es que la Alianza ha mostrado resultados valiosos (Acuerdo de libre comercio, MILA, programa de movilidad, Avance en las negociaciones en materia de medicamentos, entre otras) como producto de la integración y su capacidad de decisión, pero que aún no se termina de comprender cómo se van a materializar dichos avances respecto del desarrollo de las economías.

Lo interesante de este proceso es que la visión de economía conjunta parece prometedora, sin embargo, el crecimiento de las economías gracias a la Alianza está por verse, aún es pronto para ver si los instrumentos adoptados han traído un incremento real en el crecimiento económico, o si solo se está en presencia de las mismas economías pero sumando los resultados.

Hoy la Alianza del Pacífico se muestra como un proceso innovador, de gran envergadura que promete cambiar las relaciones económicas de los 
países que participan del proyecto y de la región en general; ahora bien, lo que resulta claro es que es un Tratado de Libre Comercio que empieza su andadura. Respecto de los demás aspectos de la integración hasta este momento se revelan como «potencial», sus logros están basados en la voluntad de los presidentes que la han formado y la historia de la integración latinoamericana ya ha mostrado la fragilidad de esta clase de compromisos.

\section{REFERENCIAS BIBLIOGRÁFICAS}

Acosta Alvarado, P. A., Más allá De La Utopía: Del Diálogo Interjudicial a La Constitucionalización Del Derecho Internacional. La Red Judicial Latinoamericana Como Prueba y Motor Del Constitucionalismo Multinivel, 2015. Tesis doctoral disponible en $<\mathrm{http}: / /$ eprints.sim.ucm.es/28119/> (última consulta, 15 junio 2016).

Ariza Villar, D. M.; Díaz Moya, M. F., Mercado Integrado Latinoamericano (MILA): ¿ Objetivos no Cumplidos?, Repositorio Institucional de la Universidad EAFIT, 2015.

BERNAL-MEZA, R., «ALIANZA DEL PACÍFICO Versus ALBA y MERCOSUR: Entre El Desafío De La Convergencia y El Riesgo De La Fragmentación De Sudamérica», Pesquisa \& Debate. Revista do Programa De Estudos Pós-Graduados Em Economia Política. vol. 26, n. ${ }^{\circ} 1$ (47), 2015.

BriceÑo Ruiz, J., «Ejes y Modelos En La Etapa Actual De La Integración Económica Regional En América Latina», Estudios Internacionales (Santiago), vol. 45, n. ${ }^{\circ} 175,2013$, pp. 9-39.

Caballero Santos, S., «Mercosur: The Role of Ideas and a More Comprehensive Regionalism», Colombia Internacional, n. ${ }^{\circ}$ 78, 2013, pp. 127-144.

Díaz-Cediel, S., «La Alianza Del Pacífico: Un Escrutinio Jurídico Al Tenor Del Derecho De Las Organizaciones Internacionales», ACDI-Anuario Colombiano De Derecho Internacional, vol. 9, 2016, pp. 347-390.

George, S., The Pacific Pumas. An Emerging Model for Emerging Markets.Washington: Bertelsmann Foundation y Global Economic Dynamics, 2014. Disponible en. $<$ http://www.bfna.org/publication/the-pacific-pumas-an-emerging-pacific-alliance-model-for-emerging-markets $>$ (última consulta, 15 junio 2016).

GonZalez-Perez, M. A., (et al)., «Pacific Alliance: Bringing Back Open Regionalism to Latin America», México y La Cuenca Del Pacifico, vol. 4, n. ${ }^{\circ}$ 11, 2015, pp. 21-51.

HERRERA, J C., «Las cláusulas de integración en las constituciones de Suramérica: 200 años después de la carta de Jamaica $\left[^{*}\right] »$, Colombia Internacional, n. ${ }^{\circ} 86$, 2016. pp. 165-192.

LÓPEZ-JACOISTE DíAZ, E., «Chapter 2. the Latin American Integration Association» en ODELLO, M.; and SEATZU, F. (eds)., Latin American and Caribbean International Institutional Law, Springer, 2015, pp. 22-42.

Ochoa Bilbao, L.; Velázquez Flores, R., «México, el MERCOSUR y la alianza del pacífico: Intereses, identidades y prioridades regionales», Miríada: Investigación En Ciencias Sociales, 2016, vol. 7, n. ${ }^{\circ}$ 11, pp. 51-64. 
Reggiardo Palacios, G., Un Nuevo Escenario Geopolitico En La Región Latinoamericana: La Creación De La Alianza Del Pacífico (2011-2015). Universidad Andina Simón Bolívar, Sede Ecuador, 2015.

Tole, J. J., «Los TLC De Estados Unidos Con Países Latinoamericanos: Un Modelo De Integración Económica «Superficial» Para El Continente Americano», Revista Derecho Del Estado, vol. 30, n. ${ }^{\circ}$ 30, 2013, pp. 251-300.

\section{INFORMES DE INSTITUCIONES Y DOCUMENTOS DIFUNDIDOS EN INTERNET}

AlianZa del Pacífico, Dirección general de comunicación social. Comunicado Conjunto De Los Países Miembros De La AP Con Motivo De La Entrada En Vigor Del Protocolo Adicional Al Acuerdo Marco, 1/05/2016.

— Protocolo Adicional Al Acuerdo Marco ¿Qué Significa? 23/02/2013. Disponible en: $<$ https://www.direcon.gob.cl/2014/02/protocolo-adicional-al-acuerdomarco-alianza-del-pacifico-que-significa/> (última consulta, 15 junio 2016).

— Temas De Trabajo/Cooperación. Disponible en: $<$ https://alianzapacifico.net/ cooperacion-cientifica-en-materia-de-cambio-climatico-en-la-alianza-del-pacifico-monitoreo-de-la-biodiversidad/> (última consulta, 15 junio 2016).

— Temas De Trabajo/cultura. Disponible en $<$ https://alianzapacifico.net/temasde-trabajo/> (última consulta, 15 junio 2016).

Casa de América - Madrid. Presentación En España De La 'Alianza Del Pacífico'. Disponible en <http://www.casamerica.es/politica/presentacion-en-espana-dela-alianza-del-pacifico $>$ (última consulta, 15 junio 2016).

Comunidad Andina de Naciones. Quienes Somos /logros. Disponible en: <http:// www.comunidadandina.org/Seccion.aspx?tipo=QU> (última consulta, 15 junio 2016).

DiARIO ABC.ES España Quiere Compartir Embajadas Con Los Países De La Alianza Del Pacífico. ed. 1/10/2013.

Diario El Tiempo. 'Alianza Del Pacifico no Es Un Proceso Contra Nadie': Santos. ed. 6/06/2012.

Diario LA NAción. El Gobierno Instó Al Mercosur a Alinearse a La Alianza Del Pacífico. ed. 5/05/2016.

El Diario.es. La Alianza Del Pacifico no Es Una Guerra En Latinoamérica, Asegura El Presidente Chile, 26/09/2013.

El Mercurio. A 500 Años De Su Descubrimiento, El Océano Pacífico Concentra La Mitad Del Comercio Global. ed. Grupo de Diarios América, 25/09/2013. Disponible en <http://vlex.com/vid/descubrimiento-paca-fico-concentra-463950982> (última consulta, 15 junio 2016).

Mercado Integrado Latinoamericano MilA. Reseña Histórica. Disponible en: $<$ http://www.mercadomila.com/home/resena $>$ (última consulta, 15 junio 2016).

Ministerio de Relaciones Exteriores de Colombia. Alianza Del Pacífico Presentó Declaración En Materia De Cambio Climático En La Conferencia De Las Partes Sobre Cambio Climático De La ONU. Disponible en: <http://www.cancilleria. 
gov.co/newsroom/news/alianza-del-pacifico-presento-declaracion-materia-cambio-climatico-la-conferencia-las $>$ (última consulta, 15 junio 2016).

Oficina Regional para AmÉRICA LATINA y el CARIBE, Formalizando La Informalidad Juvenil: Experiencias Innovadoras En América Latina y El Caribe Organización Internacional del Trabajo, 2015. Disponible en $<$ http://www.ilo.org/ wcmsp5/groups/public/---americas/---ro-lima/documents/publication/ wcms_359270.pdf $>$ (última consulta, 15 junio 2016).

- Notas Sobre Políticas Para La Formalización De Las Micro y Pequeñas Empresas. Organización Internacional del Trabajo, 2014. Disponible en $<$ http:// www.ilo.org/wcmsp5/groups/public/---americas/---ro-lima/documents/publication/wcms_318203.pdf $>$ (última consulta, 15 junio 2016).

Sistema de INFORMACIÓN SOBRE COMERCIO EXTERIOR DE LA ORGANIZACIÓN DE LOS Estados Americanos, Países: Chile, Colombia, México, Perú. Disponible en: $<$ http://www.sice.oas.org/countries_s.asp > (última consulta, 15 junio 2016).

Sistema Económico Latinoamericano y del Caribe (SELA), Parlamentarios De Perú y Chile Se Reunirán Por Alianza Del Pacífico. 29/04/2016. Disponible en: $<$ http://www.sela.org/es/prensa/servicio-informativo/2016/04/20160429/parlamentarios-de-peru-y-chile-se-reuniran-por-alianza-del-pacifico/> (última consulta, 15 junio 2016).

TITLE: Analysis and prospects of the Pacific Alliance

RESUMEN: El objetivo de este artículo es mostrar los principales aspectos que han rodeado a la Alianza del Pacífico desde su formación hasta su consolidación. Para este propósito se presenta una aproximación a los antecedentes de la integración en Latinoamérica; el segundo punto a tratar, es una exposición del contexto en el que se formó la Alianza; en tercer lugar, dar una explicación de la estructura, organización y funcionamiento de la Alianza, de acuerdo con el tratado constitutivo; en cuarto lugar, revelar los principales avances y logros que ha tenido la Alianza desde su creación y cómo sus decisiones han impactado en diferentes aspectos; en penúltimo término, señalar las perspectivas de la Alianza, las ventajas que supone para los países que participan y las particularidades de este proceso de integración. Y, por último, y a modo de conclusión, las principales fortalezas y posibles desafios que pueden ser determinantes en el éxito y consolidación de la Alianza.

Palabras Clave: Alianza del Pacífico, integración regional, Tratado de Libre Comercio TLC, integración latinoamericana.

ABSTRACT: The aim of this paper is to show the fundamental aspects about the Pacific Alliance, from its beginnings to its consolidation. In order to do this, this paper presents an approach to previous integration processes in Latin-America followed by an explanation about the context of the formation of the Alliance. In third place, it shows an exposition of the structure, organization and operation of the Alliance in accordance with its treaty of creation. In fourth place, the document exposes the main ad- 
vances and success achieved by the Alliance and the impact of its decisions in different fields. Next to last, the outlook for the Alliance, the advantages that had bring to the member states and the special features of this integration process. To conclude, the final part deals with the strengths and challenges that could be decisive in the success and final consolidation of the Pacific Alliance.

KEY WORDS: Pacific Alliance, Regional integration, Free Trade Agreement FTA, Latin-American integration.

RECIBIDO: 03.06.2016

ACEPTADO: 22.06.2016 